\title{
La edad morfológica como instrumento para la selección de talentos en escolares colombianos de
} 11 a 14 años

\section{Morphological age as an instrument for the selection of talents in Colombian schoolchildren aged 11 to 14 years}

\author{
'Universidad del Atlántico. Barranquilla, Atlántico, Colombia. \\ 2Universidad de Ciencias de la Cultura Física y el Deporte Manuel Fajardo. La Habana, Cuba. \\ ${ }^{3}$ Centro Provincial de Medicina Deportiva de Matanzas. Matanzas, Cuba. \\ *autor para correspondencia: samuelvillamarin@mail.uniatlantico.edu.co
}

Samuel Villamarín-Menza* (D); Bergelino Zaldívar-Pérez² (D); José Raúl Siret-Alfonso³ (D)

Cómo citar: Villamarín-Menza, S.; Zaldívar-Pérez, B.; Siret-Alfonso, J.R. 2021. La edad morfológica como instrumento para la selección de talentos en escolares colombianos de 11 a 14 años. Rev. Digit. Act. Fis. Deport. 7(1):e1763. http://doi.org/10.31910/ rdafd.v7.n1.2021.1763

Artículo de acceso abierto publicado por Revista Digital: Actividad Física y Deporte, bajo una licencia Creative Commons CC BY-NC 4.0 Publicación oficial de la Universidad de Ciencias Aplicadas y Ambientales U.D.C.A, Institución de Educación Superior Acreditada de Alta Calidad por el Ministerio de Educación Nacional.

Recibido: septiembre 20 de 2020 Aceptado: octubre 29 de 2020 Editado por: Álvaro José Gracia Díaz

\section{RESUMEN}

Introducción: La edad morfológica es un instrumento que permite conocer si el talento se encuentra adelantado, normal o retrasado, desde el punto de vista biológico. Objetivo: Determinar si en un grupo de niños colombianos de 11 a 14 años la presencia de diferencias significativas, entre su edad decimal y la morfológica. Metodología: Es un estudio descriptivo y transversal, con 332 escolares. Laedad morfológica fue evaluada a través del método del Índice de Desarrollo Corporal modificado (IDCm), a partir del IDC. Se evaluaron la edad decimal, el peso, la talla, los diámetros biacromial y bicrestal, las circunferencias máximas de los muslos (niñas) y de los antebrazos (niños). Se conformaron tres grupos diferenciados, según el estado de desarrollo: atrasados, normal y acelerados. Resultados: En las niñas, de los 11 a los 14 años, se encontraronvalores en promedio, desde 10,84 hasta 15,74 años. En los niños, los valores oscilaron en promedio desde 10,55 hasta 17,17 años. Conclusión: No se hallaron diferencias significativas $(p>0,05)$ en la edad morfológica, entre los escolares de una misma edad cronológica.

Palabras clave: Edad morfológica; Edad decimal; Selección de talentos; Escolares de 11 a 14 años.

\section{ABSTRACT}

Introduction: Morphological age is an instrument that allows knowing, yes, talent is advanced, normal or retarded from the biological point of view. Objective: To determine, yes, in a group of Colombian children 
aged 11 to 14 years the presence of significant differences between their decimal and morphological age. Methodology: It is a descriptive and cross-sectional study with 332 schoolchildren. Morphological age was evaluated through the modified Body Development Index (IDCm), based on the IDC. Decimal age, weight, height, biacromial and bicrestal diameters, maximum circumferences of the thighs (girls) and forearms (boys) were evaluated. Three well-differentiated groups were made up according to the stage of development: backward, normal and accelerated. Results: In girls, from 11 to 14 years old, values were found on average from 10.84 to 15.74 years. In children, the values ranged on average from 10.55 to 17.17 years. No significant differences ( $p>0.05)$ were found in morphological age among schoolchildren of the same chronological age.

Keywords: Morphological age; Decimal age; Talent selection; Schoolchildren from 11 to 14 years old.

\section{INTRODUCCIÓN}

El desarrollo biológico es un indicador que debe ser considerado en los procesos de la educación física, de la formación deportiva y de preparación de jóvenes deportistas. Las diferencias en el crecimiento y la maduración de los niños provocan distintas características estructurales y funcionales en los órganos y sistemas y, como consecuencia, se manifiestan de manera diferente las capacidades y las habilidades motrices, para responder a los esfuerzos cotidianos y las exigencias del deporte. El objetivo de la investigación fue determinar si en estos escolares colombianos, de 11 a 14 años, se presentan diferencias entre la edad morfológica y la decimal.

La selección de talentos en Colombia, se basa en el Plan Decenal del Deporte, la Recreación, la Educación Física y la Actividad Física para el Desarrollo Humano, la Convivencia y la Paz 2009 - 2019, que indica que el sistema educativo escolar es el semillero natural para la detección temprana de talentos y para identificar, organizar y articular, las reservas estratégicas al deporte de alto rendimiento. Se hace por selección natural, con los campeones de los juegos intercolegiados, como los posibles talentos.
Diferentes autores indican la edad biológica como un parámetro para seleccionar los niños y los jóvenes. Pancorbo (2002) indica que es importante conocer, en la infancia, la edad biológica de cada talento deportivo. Es una variable que permite conocer si el talento se encuentra adelantado, normal o retrasado, desde el punto de vista biológico. La edad biológica, se evalúa por edad ósea, edad morfológica y caracteres sexuales secundarios, además de la predicción de la estatura.

Otros métodos novedosos para evaluar la edad biológica son los siguientes. Ashanin et al. (2018) proponen la relación peso-altura, dado que la longitud y la masa del cuerpo determinan los procesos de organización estructural, lo que establece la velocidad de crecimiento. Malina et al. (2019), las biobandas, definidas por el porcentaje de la altura adulta, predicha en el momento de la observación, es útil para identificar y desarrollar talento; facilita la adaptación de la variación de madurez, al evaluar el talento de un individuo y proporciona un entorno apropiado y desafíos en los que los individuos se pueden desarrollar, de manera óptima.

Según Astrand \& Rodahl (1992), el crecimiento adolescente afecta el desempeño físico, por ello, no se deben agrupar los niños por la edad cronológica en una clase. Un desarrollador temprano hace que padres y entrenador sobrestimen su talento; en poco tiempo, otros pueden alcanzarlo, demostrando que su éxito se debió solo a la maduración temprana. El desarrollo del código genético puede tener más impacto sobre el desempeño físico que el medio ambiente y el estilo de vida, pero un cambio en el estilo de vida puede modificar la edad biológica, a cualquier edad.

Para Martín et al. (2001), la edad biológica designa el estado de desarrollo y la capacidad funcional del organismo en la respectiva edad cronológica. En el caso de la detección precoz de talentos, si los retrasados o desarrollados normalmente alcanzan o incluso superaran a los adelantados al terminar la fase de crecimiento, no se puede responder con seguridad. En deportes, donde el rendimiento depende de la condición física, se da preferencia a los acelerados.

Según Platonov (2001), las diferencias de desarrollo de los niños de la misma edad dependen de los ritmos 
de maduración sexual. Al seleccionar niños con desarrollo biológico acelerado, posteriormente, pierden la ventaja y desertan; más éxito obtienen los niños con un desarrollo normal o retrasado, que a los 16 - 17 años presentan un brusco incremento de las capacidades. Entre los que alcanzan la maestría deportiva internacional, el $20 \%$ tiene desarrollo acelerado; los más altos logros en este aspecto, con frecuencia, los muestran los deportistas con un desarrollo retrasado.

Para Suslov (2008), cuando el desarrollo de un niño es acelerado, la adolescencia comienza antes y rápidamente llega a su fin. Los niños con adolescencia tardía, se consideran de desarrollo retardado; a la edad de 18 años pueden sobrepasar a los de desarrollo acelerado y muestran un mejor nivel de preparación y resultados. Los atletas pueden tener diferencias hasta tres años con respecto a la edad cronológica; los 12 años, el rango de edad biológica real, se puede ubicar entre los $9-15$ años.

Para Weineck (2012), los niños maduros reflejan hasta 5 años más, algunos a los 10 u 11 años tienen talla alta y parecen talentos, pero es por la dinámica de crecimiento. En los que presentan una maduración precoz, se manifiestan los efectos de la testosterona, estrógenos u hormona de crecimiento, que aumenta la entrenabilidad y propician la óptima recuperación, lo que hace pensar que son talentos. Los que entran temprano en la pubertad son más rápidos, resistentes y fuertes. El momento del crecimiento es difícil de prever y no hay indicador que asegure que es un proceso estable.

El diagnóstico del talento, según Hohmann et al. (2005), identifica a los deportistas jóvenes, cuyo rendimiento de competición o características específicas del deporte están por encima de la media de la población. La pregunta es, ¿cuán grande debe ser la separación sobre la media? Estos autores citan a Ljach (1998), quien informó que la selección de talentos en Rusia, se utilizaba en criterio de dos desviaciones estándar (el mejor, 2,28\%). Kovar (1981) promovió, para la selección de talentos, con al menos tres desviaciones estándar (el mejor, $0,13 \%$ ) y Matsudo (1996) propuso cuatro deviaciones estándar por encima (el mejor, 0,0034\%).

La selección deportiva, según Volkov \& Filin (1988), es un sistema organizativo metodológico, que incluye métodos pedagógicos, psicológicos, sociológicos y médicos biológicos de investigación, con base, en los cuales, se detectan las capacidades de los niños y los jóvenes, para especializarse en un deporte. La selección deportiva, se basa en el conocimiento de las características modelo que poseen los mejores deportistas y cómo se forman las características en cada etapa.

La identificación y el desarrollo del talento son dos etapas de un mismo proceso. En palabras de Platonov \& Bulátova (2015), la selección deportiva es el proceso de búsqueda de las personas más dotadas, capaces de lograr altos resultados en un deporte y diferencian, en concepto de orientación deportiva, como la dirección más prospectiva en el camino hacia la maestría deportiva, basada en el estudio de las aptitudes y las capacidades, particularidades individuales y maestría.

La selección es específica para cada deporte. Según Siris et al. (1988), en los velocistas, se hace con base en los resultados de los test; sin embargo, al cabo de 2 o 3 años, los resultados de los mejores no son tan promisorios y ceden ante sus coetáneos. Las mismas pruebas y normas para niños con diferente desarrollo, pero entre los 12 y 13 años, hay unos con desarrollo acelerado y otros con ritmo retardado; esto obedece a las diferencias en el desarrollo físico y al nivel de preparación física. Pancorbo (2002) observó que la estatura es mayor en los grupos de deportistas que tienen mayor élite deportiva. En general, los campeones son de mayor estatura; el promedio de los nadadores de la olimpiada de Moscú fue superior a la de Montreal en ambos sexos y en cada ciclo olímpico, se incrementa.

Zatsiorski (1989) escribe que el pronóstico del talento deportivo, se puede realizar sobre la base de la estabilidad de los indicadores o de las influencias hereditarias. ¿Cuán estables son las características del niño en el proceso de desarrollo? Si a los alumnos del primer grado se les mide estatura, carrera de $30 \mathrm{~m}$, entre otros, ¿los niños que ocupan los primeros lugares, seguirán entre los primeros, si se miden dentro de 10 años? y ċlos más altos seguirán siendo los de más estatura o los alcanzarán aquellos que, en primer grado, se encontraban entre los más pequeños?

Al éxito, se llega por diferentes caminos. Epstein (2014) menciona dos saltadores de altura: Holm, campeón Olímpico en Atenas 2004, con 2,40m, tras 20 
años de entrenamiento, con solo $1,80 \mathrm{~m}$ de estatura y Thomas, quien en un año de entrenamiento logró 2,35 y el campeonato del mundo en Osaka 2007. La varianza en el rendimiento de las personas depende de los genes, de la práctica o algo más; aunque la práctica acumulada por 10.000 horas debería explicar la mayor parte de toda la varianza, esto no sucede; los estudios muestran que la varianza explicada por la práctica, es baja.

Según Elferink-Gemser (2013), aunque el camino hacia la cima es largo, el tiempo que los atletas talentosos tienen para aumentar su rendimiento deportivo es corto. Los elementos más importantes durante el camino hacia la cima son la maduración, el aprendizaje y el entrenamiento (cita a Malina et al. 2000; Starkes, 2000). Para realizar una identificación y adecuado y pertinente desarrollo del talento, con correlación al momento y al ritmo de un crecimiento acelerado, no deberían desempeñar un papel crucial en la selección de atletas talentosos (cita a Malina \& Rogol, 2011).

\section{MATERIALES Y MÉTODOS}

Se trata de un estudio descriptivo y transversal, de enfoque cuantitativo. Se estudiaron 148 niñas y 184 niños, para un total de 332 escolares, de 11 a 14 años de edad, que estudian en la Institución Educativa Francisco de Paula Santander, del Municipio de Galapa, Atlántico. La muestra aleatoria de escolares fue seleccionada de los grados $6^{\circ}$ a $9^{\circ}$, cumplían con los criterios de buena salud, edad, sexo y consentimiento informado (Polit \& Hungler, 2000). Las mediciones, se realizaron en el horario de clase de educación física, con la colaboración de los docentes, antes de iniciar la clase.

La edad biológica fue evaluada a través del método del Índice de Desarrollo Corporal modificado (IDCm), propuesto por Siret et al. (1991), a partir del IDC, diseñado por Wutscherk (1974). Se trata de un método no invasivo (antropométrico), para valorar la edad morfológica, mediante el IDCm; se evaluó, de cada sujeto, la edad decimal, el peso, la talla, los diámetros biacromial y bicrestal, las circunferencias máximas de los muslos (niñas) y de los antebrazos (niños); las mediciones fueron realizadas, según el protocolo de la ISAK: Stewart et al. (2011). EI IDCm, se calculó por las siguientes ecuaciones:
Sexo femenino: IDCm $=((0.5(\mathrm{DBA}+\mathrm{DBC})(0.5(\mathrm{CMD}+\mathrm{CMI}) \pm \mathrm{FC}))$ (Talla $\left.(\mathrm{cm})^{* 10}\right)$

Sexo masculino: IDCm $=((0.5(D B A+D B C)(C A D+C A l) \pm F C))$ (Talla $\left.(\mathrm{cm})^{*} 10\right)$

Dónde: $\mathrm{DBA}=$ diámetro biacromial; $\mathrm{DBC}=$ diámetro bicrestal; CMD y $\mathrm{CMI}=$ circunferencia máxima de muslo derecho e izquierdo; CAD y CAI= circunferencia máxima de antebrazo derecho e izquierdo; $F C=$ factor de corrección, que depende del Índice de Rohrer y el sexo de sujeto y se obtiene mediante las expresiones:

Sexo femenino: $F C=-14.8768$ (índice de Rohrer) $+18,4472$

Sexo masculino: $F C=-16.0735$ (índice de Rohrer) +18.1653

Índice de Rohrer: IR= (Peso corporal (gr)*100)

Talla $^{3}(\mathrm{~cm})$

Las ecuaciones predictivas para determinar la edad morfológica, son:

Sexo femenino: Edad Morfológica=0,4015*Edec+9,5469*IDCm-0,5586 Sexo masculino: Edad Morfológica $=0,5156 *$ Edec+13,4607*IDCm-4,1461

El IDCm tiene, como base de estimación de la madurez biológica individual, las variaciones de las proporciones corporales y el desarrollo en la adolescencia. EI IDCm refleja valores comprendidos entre 0,50 hasta 1,00 y más en la adolescencia, a medida que completa la maduración biológica del estado adulto. La edad morfológica tiene un alto grado de correlación con la edad ósea, medida por el método radiológico de la mano izquierda de Tanner - Whitehouse (TW2), valorado por Siret et al. (1991), en 0,89, para varones y 0,82, para mujeres. Con los resultados es posible clasificar los jóvenes, según su desarrollo: atrasado, normal y acelerado. Se realizó un análisis estadístico descriptivo y un análisis de varianza, para hallar diferencias significativas ( $p>0,05)$, entre los tres grupos, usando los programas Excel 2010 y el IBM SPSS Statistics 20. La hipótesis que se quería probar fue: Hipótesis Nula: el promedio de edad morfológica en los tres grupos es igual, con un $95 \%$ de confiabilidad.

\section{RESULTADOS}

Los escolares, se clasificaron por la edad decimal y sexo, obteniendo cuatro grupos de niñas y niños. Luego, se realizó un análisis estadístico, para dividir cada grupo de edad, con base en la edad morfológica y se obtuvieron tres subgrupos: 1) Desarrollo normal (norma), 2) Desarrollo atrasado (inferior a la norma) 
y 3) Desarrollo acelerado (superior a la norma), a partir de la media y una desviación estándar. En las niñas y niños de 11 a 14 años, se halló que la edad morfológica tiene un menor promedio en el grupo de desarrollo atrasado y el mayor valor promedio, en el de desarrollo acelerado; se tomó esta variable como referencia y, a partir de ella, se clasifican los escolares y se observa cómo se comportan las demás variables estudiadas.

En las niñas de 11 años, la edad morfológica presenta una diferencia de 2,94 años, entre el grupo retrasado y el acelerado. El Índice de Desarrollo Corporal modificado (IDCm) evoluciona del grupo desarrollo atrasado, con un valor de 0,71 al grupo acelerado, con un valor de 1,0. El indicador de peso presenta $19 \mathrm{~kg}$ de diferencia entre los tres grupos y la talla $10 \mathrm{~cm}$ de diferencia. El Índice de Masa Corporal (IMC) crece del grupo desarrollo atrasado al grupo desarrollo acelerado, lo que evidencia que, en un mismo grupo de edad cronológica, pueden existir sujetos con características antropométricas y de desarrollo físico totalmente distintos, con 7 puntos de diferencia. El porcentaje de grasa, a esta edad, también progresa del grupo desarrollo atrasado al grupo acelerado, dejando una diferencia de 11 puntos, entre ellos. Un indicador importante en los corredores de velocidad es el índice córmico, donde también crecen los valores del grupo de desarrollo atrasado al grupo desarrollo acelerado (Tabla 1).

Tabla 1. Estadística descriptiva de las escolares niñas de 11 años.

\begin{tabular}{|l|l|c|c|c|c|c|c|c|}
\hline \multirow{4}{*}{$\begin{array}{l}\text { Desarrollo } \\
\text { Atrasado }\end{array}$} & Estadígrafo & $\begin{array}{c}\text { Edad } \\
\text { morfológica }\end{array}$ & Peso & Talla & IMC & $\begin{array}{c}\text { Índice } \\
\text { córmico }\end{array}$ & IDCm & $\begin{array}{c}\text { \% } \\
\text { Grasa }\end{array}$ \\
\cline { 2 - 9 } & Promedio & 10,83 & 29,67 & 1,41 & 14,86 & 50,51 & 0,71 & 16,85 \\
\cline { 2 - 9 } & D. estándar & 0,26 & 1,75 & 0,04 & 0,33 & 1,37 & 0,03 & 1,87 \\
\cline { 2 - 9 } & C. variación & 0,02 & 0,06 & 0,03 & 0,02 & 0,03 & 0,04 & 0,11 \\
\cline { 2 - 9 } & Mínimo & 10,31 & 27,00 & 1,36 & 14,39 & 48,44 & 0,66 & 14,56 \\
\cline { 2 - 9 } & Máximo & 11,02 & 31,00 & 1,46 & 15,16 & 51,77 & 0,73 & 19,25 \\
\hline \multirow{4}{*}{$\begin{array}{l}\text { Nesarrollo } \\
\text { Normal }\end{array}$} & Promedio & 11,93 & 38,48 & 1,49 & 17,30 & 51,11 & 0,82 & 20,99 \\
\cline { 2 - 9 } & D. estándar & 0,53 & 6,07 & 0,06 & 1,78 & 1,08 & 0,05 & 4,16 \\
\cline { 2 - 9 } & Cf. variación & 0,04 & 0,16 & 0,04 & 0,10 & 0,02 & 0,06 & 0,20 \\
\cline { 2 - 9 } & Mínimo & 11,07 & 30,00 & 1,36 & 15,01 & 49,32 & 0,74 & 14,01 \\
\cline { 2 - 9 } & Máximo & 12,83 & 50,00 & 1,62 & 21,36 & 53,38 & 0,91 & 31,94 \\
\hline \multirow{4}{*}{$\begin{array}{l}\text { Desarrollo } \\
\text { Acelerado }\end{array}$} & Promedio & 13,77 & 48,00 & 1,51 & 21,02 & 51,47 & 1,00 & 27,83 \\
\cline { 2 - 9 } & D. estándar & 0,73 & 5,39 & 0,06 & 1,14 & 0,78 & 0,08 & 4,51 \\
\cline { 2 - 9 } & C. variación & 0,05 & 0,11 & 0,04 & 0,05 & 0,02 & 0,08 & 0,16 \\
\cline { 2 - 7 } & Míno & 13,24 & 43,00 & 1,45 & 20,26 & 50,34 & 0,95 & 22,67 \\
\cline { 2 - 9 } & Máximo & 15,04 & 56,00 & 1,56 & 23,01 & 52,29 & 1,14 & 33,77 \\
\hline
\end{tabular}

En las niñas de 12 años, los resultados de la edad morfológica muestran una diferencia de 2,89 años entre el grupo con desarrollo retrasado y desarrollo acelerado. El IDCm varía entre 0,73 y 1,03 del grupo atrasado al grupo acelerado. En el peso son $17 \mathrm{~kg}$ de diferencia entre los tres grupos y en la talla, $5 \mathrm{~cm}$ de diferencia. El IMC crece del grupo desarrollo atrasado al grupo desarrollo acelerado, lo que evidencia que, en un mismo grupo de edad cronológica, pueden existir sujetos con características distintas, con 6 puntos de diferencia. El porcentaje de grasa, también progresa del grupo atrasado al grupo acelerado, dejando una diferencia de 9 puntos, entre ellos. Con el índice córmico, también crecen los valores del grupo atrasado, con referencia al grupo acelerado (Tabla 2). 
Tabla 2. Estadística descriptiva de las escolares niñas de 12 años

\begin{tabular}{|l|l|c|c|c|c|c|c|c|}
\hline \multirow{4}{*}{$\begin{array}{l}\text { Desarrollo } \\
\text { Atrasado }\end{array}$} & Estadígrafo & $\begin{array}{c}\text { Edad } \\
\text { morfológica }\end{array}$ & Peso & Talla & IMC & $\begin{array}{c}\text { Índice } \\
\text { córmico }\end{array}$ & IDCm & $\begin{array}{c}\text { \% } \\
\text { Grasa }\end{array}$ \\
\cline { 2 - 9 } & Promedio & 11,46 & 34,71 & 1,50 & 15,44 & 50,73 & 0,73 & 17,34 \\
\cline { 2 - 9 } & D. estándar & 0,17 & 4,03 & 0,09 & 2,26 & 1,33 & 0,02 & 3,16 \\
\cline { 2 - 9 } & C. variación & 0,01 & 0,12 & 0,06 & 0,15 & 0,03 & 0,03 & 0,18 \\
\cline { 2 - 9 } & Mínimo & 11,13 & 29,00 & 1,39 & 13,33 & 48,43 & 0,69 & 12,73 \\
\cline { 2 - 9 } & Máximo & 11,62 & 39,00 & 1,65 & 20,19 & 52,88 & 0,76 & 22,24 \\
\hline \multirow{4}{*}{$\begin{array}{l}\text { Desarrollo } \\
\text { normal }\end{array}$} & Promedio & 12,62 & 39,81 & 1,50 & 17,63 & 51,27 & 0,85 & 21,81 \\
\cline { 2 - 9 } & D. estándar & 0,48 & 4,19 & 0,06 & 1,42 & 1,11 & 0,05 & 3,25 \\
\cline { 2 - 9 } & C. variación & 0,04 & 0,11 & 0,04 & 0,08 & 0,02 & 0,06 & 0,15 \\
\cline { 2 - 9 } & Mínimo & 11,91 & 32,00 & 1,41 & 15,01 & 49,03 & 0,77 & 15,47 \\
\cline { 2 - 9 } & Máximo & 13,57 & 47,00 & 1,63 & 20,12 & 53,64 & 0,96 & 28,10 \\
\hline \multirow{4}{*}{$\begin{array}{l}\text { Desarrollo } \\
\text { Acelerado }\end{array}$} & Promedio & 14,35 & 51,22 & 1,55 & 21,43 & 51,03 & 1,03 & 26,90 \\
\cline { 2 - 9 } & D. estándar & 0,38 & 4,58 & 0,05 & 1,46 & 1,69 & 0,04 & 4,37 \\
\cline { 2 - 9 } & C. variación & 0,03 & 0,09 & 0,03 & 0,07 & 0,03 & 0,04 & 0,16 \\
\cline { 2 - 5 } & 13,92 & 45,00 & 1,47 & 19,74 & 48,34 & 1,00 & 19,56 \\
\cline { 2 - 9 } & Máximo & 15,23 & 59,00 & 1,65 & 24,77 & 53,06 & 1,12 & 34,69 \\
\hline
\end{tabular}

En las niñas de 13 años, los resultados de la edad morfológica muestran diferencias entre los tres grupos de 3,54 años. El IDCm presenta valores desde 0,75 , en el grupo atrasado hasta 1,12, en el grupo acelerado. Las variables de peso y talla tienen diferencias; en el peso, la diferencia entre el grupo atrasado y el grupo acelerado es de $18 \mathrm{~kg}$ y en la talla, la diferencia alcanza los $6 \mathrm{~cm}$. El IMC tiene una diferencia de 7 puntos entre los escolares de esta edad. El porcentaje de grasa, la diferencia es de 10 puntos, entre los tres grupos. El índice córmico muestra valores muy cercanos de 52,35 a 52,40, entre el grupo atrasado y el grupo acelerado (Tabla 3 ).

Tabla 3. Estadística descriptiva de las escolares niñas de 13 años

\begin{tabular}{|l|l|c|c|c|c|c|c|c|}
\hline \multirow{4}{*}{$\begin{array}{l}\text { Desarrollo } \\
\text { atrasado }\end{array}$} & Estadígrafo & $\begin{array}{c}\text { Edad } \\
\text { morfológica }\end{array}$ & Peso & Talla & IMC & $\begin{array}{c}\text { Índice } \\
\text { córmico }\end{array}$ & IDCm & $\begin{array}{c}\% \\
\text { Grasa }\end{array}$ \\
\cline { 2 - 9 } & Promedio & 12,10 & 36,00 & 1,50 & 15,99 & 52,35 & 0,75 & 17,68 \\
\cline { 2 - 9 } & D. estándar & 0,46 & 3,16 & 0,06 & 0,96 & 2,11 & 0,04 & 2,81 \\
\cline { 2 - 9 } & C. variación & 0,04 & 0,09 & 0,04 & 0,06 & 0,04 & 0,05 & 0,16 \\
\cline { 2 - 9 } & Mínimo & 11,16 & 32,00 & 1,44 & 15,11 & 49,69 & 0,68 & 14,62 \\
\cline { 2 - 9 } & Máximo & 12,50 & 41,00 & 1,61 & 17,84 & 56,45 & 0,81 & 22,18 \\
\hline \multirow{4}{*}{$\begin{array}{l}\text { Desarrollo } \\
\text { Normal }\end{array}$} & Promedio & 13,64 & 45,85 & 1,55 & 19,17 & 51,71 & 0,92 & 24,02 \\
\cline { 2 - 9 } & D. estándar & 0,64 & 5,08 & 0,05 & 1,77 & 1,43 & 0,07 & 4,30 \\
\cline { 2 - 9 } & C. variación & 0,05 & 0,11 & 0,03 & 0,09 & 0,03 & 0,07 & 0,18 \\
\cline { 2 - 9 } & Mínimo & 12,56 & 35,00 & 1,45 & 16,23 & 48,43 & 0,81 & 18,34 \\
\cline { 2 - 9 } & Máximo & 14,83 & 57,00 & 1,65 & 22,75 & 54,48 & 1,04 & 37,13 \\
\hline \multirow{4}{*}{$\begin{array}{l}\text { Desarrollo } \\
\text { Acelerado }\end{array}$} & Promedio & 15,64 & 54,29 & 1,56 & 22,22 & 52,40 & 1,12 & 27,95 \\
\cline { 2 - 9 } & D. estándar & 0,41 & 3,59 & 0,04 & 1,26 & 1,39 & 0,05 & 5,45 \\
\cline { 2 - 9 } & C. variación & 0,03 & 0,07 & 0,02 & 0,06 & 0,03 & 0,04 & 0,19 \\
\cline { 2 - 5 } & 15,02 & 49,00 & 1,50 & 20,40 & 50,47 & 1,05 & 20,11 \\
\cline { 2 - 8 } & Máximo & 16,21 & 58,00 & 1,61 & 24,62 & 55,00 & 1,17 & 35,84 \\
\hline
\end{tabular}


Las niñas de 14 años, con mayor maduración, presentan una edad morfológica, que varía del grupo atrasado al grupo acelerado, en 2,43 años. Se observan diferentes valores del IDCm, entre 0,85 y 1,09 , entre el grupo atrasado y el grupo acelerado. EI peso y la talla siguen un comportamiento ascendente del grupo atrasado al grupo acelerado, con valores entre 44 y $59 \mathrm{~kg}$. La talla en esta edad muestra una tendencia inversa con el mayor valor en el grupo atrasado, con $1,61 \mathrm{~cm}$ y $1,58 \mathrm{~cm}$, en el grupo acelerado. EI IMC crece del grupo atrasado al grupo acelerado, con 7 puntos de diferencia. El porcentaje de grasa muestra un valor creciente a esta edad, con una diferencia de 11 puntos, entre los tres grupos. El índice córmico muestra valores entre 50,38 y 52,63, entre los tres grupos (Tabla 4).

Tabla 4. Estadística descriptiva de las escolares niñas de 14 años

\begin{tabular}{|l|l|r|r|r|r|r|r|r|}
\hline \multirow{4}{*}{$\begin{array}{l}\text { Desarrollo } \\
\text { Atrasado }\end{array}$} & Estadígrafo & $\begin{array}{c}\text { Edad } \\
\text { morfológica }\end{array}$ & Peso & Talla & \multicolumn{1}{|c|}{ IMC } & $\begin{array}{c}\text { Índice } \\
\text { córmico }\end{array}$ & IDCm & $\begin{array}{c}\text { \% } \\
\text { Grasa }\end{array}$ \\
\cline { 2 - 9 } & Promedio & 13,31 & 44,00 & 1,61 & 17,01 & 50,38 & 0,85 & 20,88 \\
\cline { 2 - 9 } & D. estándar & 0,07 & 2,65 & 0,02 & 0,88 & 2,42 & 0,01 & 2,24 \\
\cline { 2 - 10 } & C. variación & 0,01 & 0,06 & 0,01 & 0,05 & 0,05 & 0,01 & 0,11 \\
\cline { 2 - 9 } & Mínimo & 13,24 & 41,00 & 1,60 & 16,02 & 47,85 & 0,85 & 18,34 \\
\cline { 2 - 9 } & Máximo & 13,37 & 46,00 & 1,63 & 17,69 & 52,66 & 0,86 & 22,55 \\
\hline \multirow{4}{*}{$\begin{array}{l}\text { Desarrollo } \\
\text { Normal }\end{array}$} & Promedio & 14,00 & 46,00 & 1,57 & 18,60 & 50,60 & 0,92 & 24,70 \\
\cline { 2 - 9 } & D. estándar & 0,55 & 4,65 & 0,05 & 1,33 & 2,08 & 0,06 & 4,67 \\
\cline { 2 - 9 } & C. variación & 0,04 & 0,10 & 0,03 & 0,07 & 0,04 & 0,07 & 0,19 \\
\cline { 2 - 9 } & Mínimo & 13,42 & 36,00 & 1,49 & 15,68 & 43,71 & 0,85 & 17,18 \\
\cline { 2 - 9 } & Máximo & 15,18 & 54,00 & 1,65 & 21,23 & 53,70 & 1,06 & 35,30 \\
\hline \multirow{4}{*}{$\begin{array}{l}\text { Desarrollo } \\
\text { Acelerado }\end{array}$} & Promedio & 15,74 & 59,83 & 1,58 & 24,07 & 52,63 & 1,09 & 31,77 \\
\cline { 2 - 9 } & D. estándar & 0,25 & 7,63 & 0,10 & 1,42 & 1,26 & 0,04 & 3,93 \\
\cline { 2 - 9 } & C. variación & 0,02 & 0,13 & 0,06 & 0,06 & 0,02 & 0,04 & 0,12 \\
\cline { 2 - 9 } & Mínimo & 15,46 & 50,00 & 1,41 & 21,37 & 51,28 & 1,03 & 24,80 \\
\cline { 2 - 9 } & Máximo & 16,06 & 69,00 & 1,69 & 25,24 & 54,19 & 1,14 & 36,15 \\
\hline
\end{tabular}

Resultados en los niños. A los 11 años, la edad morfológica muestra una variación de 1,37 años, entre los tres grupos. EI IDCm presenta valores que oscilan entre 0,62 , en el grupo atrasado y 0,72 , en el grupo acelerado. El peso corporal de los escolares oscila entre 31 y $33 \mathrm{~kg}$, mientras que la talla va de los 1,41 , en el grupo atrasado a 1,45 , en el grupo acelerado. EI IMC no se caracteriza por grandes diferencias entre los tres grupos, porque sus valores varían solo entre 15 y 16 puntos. El porcentaje de grasa, tampoco registra grandes variaciones, oscilando entre 13 y 16 puntos. El índice córmico alcanza valores muy homogéneos, entre 49,55 , en el grupo acelerado y 50,47, en el grupo atrasado (Tabla 5).

Tabla 5. Estadística descriptiva de los escolares niños de 11 años

\begin{tabular}{|l|l|c|c|c|c|c|c|c|}
\hline \multirow{4}{*}{$\begin{array}{l}\text { Desarrollo } \\
\text { atrasado }\end{array}$} & Estadígrafo & $\begin{array}{c}\text { Edad } \\
\text { morfológica }\end{array}$ & Peso & Talla & IMC & $\begin{array}{c}\text { Índice } \\
\text { córmico }\end{array}$ & IDCm & $\begin{array}{c}\% \\
\text { Grasa }\end{array}$ \\
\cline { 2 - 9 } & Promedio & 10,55 & 31,29 & 1,41 & 15,57 & 50,47 & 0,65 & 13,06 \\
\cline { 2 - 9 } & D. estándar & 0,17 & 6,18 & 0,07 & 1,64 & 2,56 & 0,01 & 4,31 \\
\cline { 2 - 9 } & C. variación & 0,02 & 0,20 & 0,05 & 0,11 & 0,05 & 0,02 & 0,33 \\
\cline { 2 - 9 } & Mínimo & 10,35 & 27,00 & 1,36 & 14,18 & 45,16 & 0,63 & 7,91 \\
\cline { 2 - 9 } & Máximo & 10,77 & 45,00 & 1,55 & 18,73 & 52,45 & 0,66 & 20,48 \\
\hline \multirow{4}{*}{$\begin{array}{l}\text { Desarrollo } \\
\text { normal }\end{array}$} & Promedio & 11,42 & 31,71 & 1,43 & 15,37 & 50,05 & 0,69 & 13,45 \\
\cline { 2 - 9 } & D. estándar & 0,33 & 4,60 & 0,07 & 1,24 & 1,28 & 0,04 & 3,19 \\
\cline { 2 - 9 } & C. variación & 0,03 & 0,14 & 0,05 & 0,08 & 0,03 & 0,05 & 0,24 \\
\cline { 2 - 9 } & Mínimo & 10,81 & 22,00 & 1,27 & 13,60 & 47,02 & 0,61 & 8,64 \\
\cline { 2 - 9 } & Máximo & 11,80 & 41,00 & 1,58 & 19,50 & 52,41 & 0,75 & 19,74 \\
\hline \multirow{4}{*}{$\begin{array}{l}\text { Desarrollo } \\
\text { acelerado }\end{array}$} & Promedio & 11,92 & 33,67 & 1,45 & 16,06 & 49,55 & 0,72 & 16,64 \\
\cline { 2 - 9 } & D. estándar & 0,04 & 3,72 & 0,05 & 1,18 & 1,13 & 0,02 & 4,89 \\
\cline { 2 - 9 } & C. variación & 0,00 & 0,11 & 0,03 & 0,07 & 0,02 & 0,03 & 0,29 \\
\cline { 2 - 8 } & Mínimo & 11,87 & 29,00 & 1,40 & 14,59 & 47,84 & 0,70 & 10,04 \\
\cline { 2 - 9 } & Máximo & 11,97 & 40,00 & 1,51 & 17,66 & 51,38 & 0,76 & 23,34 \\
\hline
\end{tabular}


En los niños de 12 años, la edad morfológica es muy homogénea y varía en 0,78 años, entre los tres grupos. De la misma manera, el IDCm presenta pequeñas variaciones, que reflejan el desarrollo de los escolares, con valores entre 0,73 y 0,78 . En el peso, los valores del peso oscilan de $33 \mathrm{~kg}$, en grupo atrasado a $39 \mathrm{~kg}$, en grupo acelerado. La talla entre varía de $1,46 \mathrm{~cm}$, en grupo atrasado a $1,53 \mathrm{~cm}$, en el grupo norma. El IMC muestra valores entre 15 y 17 puntos, entre los tres grupos. El porcentaje de grasa está entre 13 y 16 , en los tres grupos. El índice córmico exhibe un valor similar de 50, para los tres grupos, es decir, se trata de un grupo sin mayor variabilidad, en la mayoría de sus índices (Tabla 6).

Tabla 6. Estadística descriptiva de los escolares niños de 12 años

\begin{tabular}{|l|l|c|c|c|c|c|c|c|}
\hline \multirow{4}{*}{$\begin{array}{l}\text { Desarrollo } \\
\text { atrasado }\end{array}$} & Estadígrafo & $\begin{array}{c}\text { Edad } \\
\text { morfológica }\end{array}$ & Peso & Talla & IMC & $\begin{array}{c}\text { Índice } \\
\text { córmico }\end{array}$ & IDCm & $\begin{array}{c}\% \\
\text { Grasa }\end{array}$ \\
\cline { 2 - 9 } & Promedio & 12,15 & 33,22 & 1,46 & 15,58 & 50,27 & 0,73 & 13,09 \\
\cline { 2 - 9 } & D. estándar & 0,07 & 4,24 & 0,08 & 1,07 & 1,56 & 0,03 & 4,33 \\
\cline { 2 - 9 } & C. variación & 0,01 & 0,13 & 0,05 & 0,07 & 0,03 & 0,04 & 0,33 \\
\cline { 2 - 9 } & Mínimo & 12,06 & 26,00 & 1,33 & 13,55 & 47,92 & 0,70 & 9,31 \\
\cline { 2 - 9 } & Máximo & 12,24 & 41,00 & 1,60 & 17,53 & 52,63 & 0,78 & 23,27 \\
\hline \multirow{4}{*}{$\begin{array}{l}\text { Desarrollo } \\
\text { normal }\end{array}$} & Promedio & 12,54 & 38,86 & 1,53 & 16,67 & 50,08 & 0,75 & 16,24 \\
\cline { 2 - 9 } & D. estándar & 0,18 & 4,68 & 0,08 & 1,60 & 1,36 & 0,04 & 5,12 \\
\cline { 2 - 9 } & C. variación & 0,01 & 0,12 & 0,05 & 0,10 & 0,03 & 0,05 & 0,32 \\
\cline { 2 - 9 } & Mínimo & 12,26 & 31,00 & 1,43 & 14,36 & 45,86 & 0,65 & 9,38 \\
\cline { 2 - 9 } & Máximo & 12,81 & 49,00 & 1,81 & 20,36 & 53,27 & 0,81 & 29,52 \\
\hline \multirow{4}{*}{$\begin{array}{l}\text { Desarrollo } \\
\text { acelerado }\end{array}$} & Promedio & 12,93 & 39,30 & 1,51 & 17,31 & 50,47 & 0,78 & 14,35 \\
\cline { 2 - 9 } & D. estándar & 0,05 & 5,12 & 0,07 & 2,04 & 1,24 & 0,05 & 5,57 \\
\cline { 2 - 9 } & C. variación & 0,00 & 0,13 & 0,05 & 0,12 & 0,02 & 0,07 & 0,39 \\
\cline { 2 - 9 } & Mínimo & 12,80 & 32,00 & 1,43 & 13,93 & 48,98 & 0,71 & 9,23 \\
\cline { 2 - 9 } & Máximo & 12,99 & 50,00 & 1,60 & 19,91 & 52,63 & 0,85 & 23,64 \\
\hline
\end{tabular}

Los niños de 13 años muestran una edad morfológica, con una diferencia de 0,79 años, entre los tres grupos. EI IDCm solo oscila entre 0,79 y 0,81 , del grupo atrasado al grupo acelerado. El peso corporal de los escolares varía entre 39,5 y $43,88 \mathrm{~kg}$, entre el grupo atrasado y el grupo acelerado, mientras que la talla de los escolares varía entre 1,49 , en el grupo atrasado y 1,57, en el grupo norma. El IMC no tiene variaciones importantes, ubicándose en los 17 puntos, en los tres grupos. El porcentaje de grasa oscila entre 14 y 16 puntos, encontrándose un menor valor en el grupo acelerado. El índice córmico presenta valores cercanos entre 50 y 51, entre los tres grupos (Tabla 7).

Tabla 7. Estadística descriptiva de los escolares niños de 13 años

\begin{tabular}{|c|c|c|c|c|c|c|c|c|}
\hline \multirow{6}{*}{$\begin{array}{l}\text { Desarrollo } \\
\text { atrasado }\end{array}$} & Estadígrafo & $\begin{array}{c}\text { Edad } \\
\text { morfológica }\end{array}$ & Peso & Talla & IMC & $\begin{array}{c}\begin{array}{c}\text { Índice } \\
\text { córmico }\end{array} \\
\end{array}$ & IDCm & $\begin{array}{c}\% \\
\text { Grasa }\end{array}$ \\
\hline & Promedio & 13,09 & 39,50 & 1,49 & 17,75 & 51,01 & 0,79 & 16,91 \\
\hline & D. estándar & 0,05 & 4,25 & 0,08 & 1,28 & 1,21 & 0,03 & 4,88 \\
\hline & C. variación & 0,00 & 0,11 & 0,05 & 0,07 & 0,02 & 0,04 & 0,29 \\
\hline & Mínimo & 13,02 & 32,00 & 1,38 & 15,23 & 49,67 & 0,73 & 10,26 \\
\hline & Máximo & 13,17 & 45,00 & 1,60 & 19,22 & 53,33 & 0,83 & 24,81 \\
\hline \multirow{5}{*}{$\begin{array}{l}\text { Desarrollo } \\
\text { normal }\end{array}$} & Promedio & 13,47 & 43,48 & 1,57 & 17,65 & 50,60 & 0,79 & 16,84 \\
\hline & D. estándar & 0,17 & 5,78 & 0,08 & 1,49 & 1,19 & 0,04 & 5,23 \\
\hline & C. variación & 0,01 & 0,13 & 0,05 & 0,08 & 0,02 & 0,05 & 0,31 \\
\hline & Mínimo & 13,19 & 33,00 & 1,44 & 14,86 & 48,77 & 0,72 & 11,14 \\
\hline & Máximo & 13,73 & 57,00 & 1,72 & 21,11 & 52,81 & 0,86 & 29,08 \\
\hline \multirow{5}{*}{$\begin{array}{l}\text { Desarrollo } \\
\text { acelerado }\end{array}$} & Promedio & 13,88 & 43,88 & 1,56 & 17,93 & 51,19 & 0,81 & 14,53 \\
\hline & D. estándar & 0,09 & 3,64 & 0,07 & 0,81 & 1,02 & 0,03 & 3,73 \\
\hline & C. variación & 0,01 & 0,08 & 0,04 & 0,05 & 0,02 & 0,04 & 0,26 \\
\hline & Mínimo & 13,77 & 39,00 & 1,47 & 16,88 & 49,39 & 0,76 & 9,67 \\
\hline & Máximo & 14,00 & 49,00 & 1,67 & 19,44 & 52,46 & 0,85 & 22,17 \\
\hline
\end{tabular}


Los niños de 14 años muestran una edad morfológica, que varía en 3,06 años, entre los tres grupos. El IDCm oscila entre 0,83 y 1,05 , entre los grupos atrasado al acelerado. Los valores del peso corporal, se mueven en un rango de $14 \mathrm{~kg}$, entre el grupo atrasado al grupo acelerado. En la talla, se observa una variación de
$5 \mathrm{~cm}$, entre los tres grupos. El IMC varía hasta 4 puntos entre los grupos atrasado, con 18,86, al acelerado, con 22,87 . El porcentaje de grasa varía entre 18,62 y 21,25 puntos, entre los tres grupos conformados. El índice córmico, se mantiene estable, entre 50 y 51 puntos (Tabla 8)

Tabla 8. Estadística descriptiva de los escolares niños de 14 años

\begin{tabular}{|l|l|c|c|c|c|c|c|c|}
\hline & Estadígrafo & $\begin{array}{c}\text { Edad } \\
\text { morfológica }\end{array}$ & Peso & Talla & IMC & $\begin{array}{c}\text { Índice } \\
\text { córmico }\end{array}$ & IDCm & $\begin{array}{c}\% \\
\text { Grasa }\end{array}$ \\
\cline { 2 - 9 } & Promedio & 14,11 & 48,33 & 1,60 & 18,86 & 50,02 & 0,83 & 18,62 \\
\cline { 2 - 9 } $\begin{array}{l}\text { Desarrollo } \\
\text { Atrasado }\end{array}$ & D. estándar & 0,07 & 3,08 & 0,05 & 1,56 & 0,63 & 0,05 & 7,49 \\
\cline { 2 - 9 } & C. variación & 0,00 & 0,06 & 0,03 & 0,08 & 0,01 & 0,05 & 0,40 \\
\cline { 2 - 9 } & Mínimo & 14,02 & 43,00 & 1,55 & 16,38 & 49,38 & 0,79 & 10,11 \\
\cline { 2 - 9 } & Máximo & 14,19 & 52,00 & 1,67 & 20,53 & 51,20 & 0,88 & 30,99 \\
\hline \multirow{4}{*}{$\begin{array}{l}\text { Desarrollo } \\
\text { normal }\end{array}$} & Promedio & 15,02 & 49,70 & 1,61 & 19,14 & 50,81 & 0,89 & 15,37 \\
\cline { 2 - 9 } & D. estándar & 0,62 & 5,95 & 0,08 & 1,67 & 1,21 & 0,05 & 5,24 \\
\cline { 2 - 9 } & C. variación & 0,04 & 0,12 & 0,05 & 0,09 & 0,02 & 0,06 & 0,34 \\
\cline { 2 - 9 } & Mínimo & 14,08 & 37,00 & 1,41 & 16,18 & 48,02 & 0,79 & 9,45 \\
\cline { 2 - 9 } & Máximo & 16,32 & 64,00 & 1,78 & 24,97 & 53,70 & 1,02 & 37,90 \\
\hline \multirow{4}{*}{$\begin{array}{l}\text { Desarrollo } \\
\text { acelerado }\end{array}$} & Promedio & 17,17 & 62,20 & 1,65 & 22,87 & 51,51 & 1,05 & 21,25 \\
\cline { 2 - 9 } & D. estándar & 0,47 & 6,60 & 0,06 & 1,93 & 1,47 & 0,05 & 7,06 \\
\cline { 2 - 9 } & C. variación & 0,03 & 0,11 & 0,04 & 0,08 & 0,03 & 0,05 & 0,33 \\
\cline { 2 - 9 } & Míno & 16,45 & 50,00 & 1,56 & 19,90 & 49,14 & 0,95 & 16,29 \\
\cline { 2 - 6 } & Máximo & 18,05 & 73,00 & 1,75 & 25,40 & 53,85 & 1,12 & 38,85 \\
\hline
\end{tabular}

Aunque los resultados descriptivos muestran diferencias entre los tres grupos conformados, a partir de la edad morfológica, el análisis de varianza mostró que, en la mayoría de los casos, no existen diferencias estadísticamente significativas, entre las variables estudiadas $(p>0,05)$, en cada una de las edades, salvo algunas excepciones, es decir que, en general, se confirma la hipótesis nula, la cual, dice que el promedio de las variables estudiadas en los tres grupos de cada edad (desarrollo atrasado, normal y acelerado) son iguales, con el $95 \%$ de confiabilidad (Tabla 9).

Tabla 9. Análisis de varianza para determinar diferencias significativas entre los grupos de desarrollo atrasado, normal y acelerado $(p>0,05)$.

\begin{tabular}{|l|c|c|c|c|c|c|c|c|}
\hline \multirow{2}{*}{ Variables } & \multicolumn{4}{|c|}{ Niñas } & \multicolumn{4}{c|}{ Niños } \\
\cline { 2 - 10 } & $\mathbf{1 1}$ & $\mathbf{1 2}$ & $\mathbf{1 3}$ & $\mathbf{1 4}$ & $\mathbf{1 1}$ & $\mathbf{1 2}$ & $\mathbf{1 3}$ & $\mathbf{1 4}$ \\
\hline $\begin{array}{l}\text { Edad } \\
\text { morfológica }\end{array}$ & 1,32 & 8,01 & 4,92 & 2,54 & 1,65 & 1,75 & 6,56 & 1,29 \\
\hline IDCm & 1,59 & 1,70 & 8,01 & 3,63 & $0,001^{*}$ & $0,02^{*}$ & 0,40 & 1,66 \\
\hline Peso & 2,65 & 1,86 & 3,95 & 1,26 & 0,62 & $0,007^{*}$ & 0,10 & 2,9 \\
\hline Talla & $0,016^{*}$ & 0,21 & 0,056 & 0,60 & 0,66 & 0,07 & $0,04^{*}$ & 0,28 \\
\hline IMC & 1,45 & 6,9 & 5,49 & 1,92 & 0,41 & 0,074 & 0,88 & 1,62 \\
\hline Tallas sentado & $0,01^{*}$ & 0,24 & $0,043^{*}$ & 0,23 & 0,92 & 0,074 & 0,12 & $0,016^{*}$ \\
\hline $\begin{array}{l}\text { Longitud } \\
\text { pierna }\end{array}$ & 0,13 & 0,18 & 0,22 & 0,21 & 0,55 & 0,16 & $0,03^{*}$ & 0,84 \\
\hline Índice córmico & 0,34 & 0,59 & 0,44 & 0,09 & 0,59 & 0,73 & 0,40 & 0,062 \\
\hline Grasa \% & $0,003^{*}$ & 1,30 & $0,003^{*}$ & $0,002^{*}$ & 0,16 & 0,24 & 0,49 & $0,013^{*}$ \\
\hline
\end{tabular}

*Los valores con asterisco son inferiores a p>0,05. 


\section{DISCUSIÓN}

En los niños en etapa de crecimiento y de desarrollo, la edad biológica varía. Los resultados de la edad decimal y la edad morfológica muestran que existen diferencias en ambas variables; puede tener una edad decimal cercana a su edad morfológica, superior o inferior. Al clasificar los niños de 11 a 14 años, se encontró que corresponden a edad morfológica atrasada, normal y acelerada. Esto es normal entre un grupo de niños de la misma edad y sexo y se debe tener en cuenta, en las clases de educación física, de deporte y en procesos de selección de talentos, para un determinado deporte. Según Martín et al. (2001), el desarrollo individual puede diferir de los promedios, sobre todo, en la fase de la pubertad y citan a Hollmann \& Hettinger (1980), quienes hallaron que las mayores diferencias alcanzaron 3,4 años a los 13 años, para los chicos y 3 años a los 11 años, para las chicas.

Según Miló (2007), en los programas de preparación de diferentes deportes practicados en Cuba, no se contemplan indicadores que tengan en cuenta la edad biológica en la planificación de las cargas de los atletas escolares, cuando diversas investigaciones demuestran que los niños con retardo y con adelanto en su desarrollo biológico con respecto a la edad cronológica, se someten a las mismas exigencias físicas, en el proceso de entrenamiento deportivo.

Las edades cronológica y biológica no siempre marchan a la par. Los resultados de esta investigación muestran que el desarrollo biológico de los escolares de Galapa, se comportan de acuerdo con la distribución normal, aproximadamente, las $2 / 3$ partes están en el grupo normal y el resto a más o menos una desviación estándar. En las niñas, que presentan primero los cambios de la pubertad, se puede observar que las diferencias entre la edad decimal y la edad morfológica reflejan proporciones distintas en los procesos de maduración biológica. Se halló que, a los 11 años de edad cronológica, 6 niñas presentan un desarrollo atrasado, 23 normal y 5 acelerado. A los 12 años de edad, 7 niñas tienen desarrollo atrasado, 27 normal y 9 acelerado. A los 13 años de edad, 7 niñas presentan desarrollo atrasado, 27 normal y 7 acelerado. A los 14 años de edad, 3 niñas tienen desarrollo atrasado, 21 normal y 6 acelerado (Tabla 10).

Tabla 10. Número de casos edad morfológica niñas de 11 a 14 años

\begin{tabular}{|l|c|c|c|c|c|c|}
\hline $\begin{array}{c}\text { Edad } \\
\text { cronológica }\end{array}$ & $\mathbf{1 1}$ & $\mathbf{1 2}$ & $\mathbf{1 3}$ & $\mathbf{1 4}$ & Total & $\%$ \\
\hline Atrasado & 6 & $\mathbf{7}$ & $\mathbf{7}$ & 3 & 23 & 15.54 \\
\hline Normal & 23 & 27 & 27 & 21 & 98 & 66.22 \\
\hline Acelerado & 5 & 9 & 7 & 6 & 27 & 18.24 \\
\hline Total & 34 & 43 & 41 & 30 & 148 & 100 \\
\hline
\end{tabular}

En los niños, los cambios de la pubertad llegan un poco más tarde, pero siguen la misma dinámica. Las diferencias entre la edad decimal y la edad morfológica son evidentes, cuando se evalúa caso por caso. A los 11 años de edad cronológica, 7 niños presentan un desarrollo atrasado, 21 normal y 6 acelerado. A los 12 años, 9 presentan desarrollo atrasado, 28 normal y 10 acelerado. A los 13 años, 10 presentan un desarrollo atrasado, 23 normal y 8 acelerado. A los 14 años de edad, 6 presentan desarrollo atrasado, 46 normal y 10 acelerado (Tabla 11).

Tabla 11. Número de casos edad morfológica niños de 11 a 14 años

\begin{tabular}{|l|c|c|c|c|c|c|}
\hline $\begin{array}{c}\text { Edad } \\
\text { cronológica }\end{array}$ & $\mathbf{1 1}$ & $\mathbf{1 2}$ & $\mathbf{1 3}$ & $\mathbf{1 4}$ & Total & $\%$ \\
\hline Atrasado & $\mathbf{7}$ & 9 & 10 & 6 & 32 & 17.39 \\
\hline Normal & 21 & 28 & 23 & 46 & 118 & 64.13 \\
\hline Acelerado & 6 & 10 & 8 & 10 & 34 & 18.48 \\
\hline Total & 34 & 47 & 41 & 62 & 184 & 100 \\
\hline
\end{tabular}


Para Ashanin et al. (2015), el problema de la individualización en la educación física supone una descripción de los hábitos específicos para cada edad. El enfoque tradicional, se basa en los índices promedio de diferenciación y de maduración de los sistemas del organismo. Un inconveniente de este enfoque para la determinación de la edad biológica es la inconsistencia cronológica de estos indicadores en una persona, porque los criterios de desarrollo biológico no coinciden con un modelo cronológico de desarrollo físico normal.

Según Doležajová et al. (2019), se confirma que existen grandes diferencias en la edad biológica en niñas de 13 años, de casi cuatro años, se descubrieron diferencias en los indicadores físicos y en el rendimiento motor en grupos con la misma edad decimal y biológica y en niñas con maduración tardía o maduración temprana. Las jugadoras con maduración tardía logran mayores mejoras en los resultados de las pruebas y, gradualmente, igualan a las biológicamente aceleradas. Se recomienda prestar atención a jugadores de desarrollo atrasado.

Los resultados de la investigación evidencian que los escolares, en el proceso de crecimiento y desarrollo, presentan diferencias que permiten agruparlos, por lo menos, en tres grupos de desarrollo: atrasado, normal y acelerado. Este factor, se debe tener en cuenta, en los procesos de búsqueda de talentos para el deporte, porque se deben observar las características sobresalientes del niño en una edad determinada y la evolución de las capacidades durante varios años.

Como afirma Astrand (2000), la mayor variación interindividual de la edad biológica se ubica entre las edades cronológicas de 12-14 años. Como promedio, la edad de velocidad máxima de crecimiento fue a los 12 años, para las niñas (con un rango de 9,5 a 15 años) $y$, en los niños, a los 14 años (con un rango de 11 a 17 años de edad). Los resultados de esta investigación concuerdan con lo reportado en la literatura.

Las diferencias y particularidades del desarrollo biológico, se observan en las diferentes variables. Según Tittel \& Wutscherk (2000), el incremento de la talla incrementa también la masa corporal, el área de superficie corporal y el volumen corporal, así como la palanca de los músculos. La ventaja que pueden tener los atletas altos de las pruebas de pista es consecuencia de una mayor palanca en las extremidades largas. En los velocistas, la relación entre las características antropométricas y la longitud de la zancada ha sido demostrada ampliamente.

\section{CONCLUSIONES}

- Al agrupar a los escolares por la edad morfológica, se conformaron tres grupos bien diferenciados, según el desarrollo: atrasados, normal y acelerados. En las niñas, de los 11 a los 14 años, se encontraron valores, en promedio, desde 10,84 hasta 15,74 años. En los niños, los valores oscilaron en promedio desde 10,55 hasta 17,17 años. No se hallaron diferencias significativas $(p>0,05)$ en la edad morfológica, entre los escolares de una misma edad cronológica.

- De los 11 a los 14 años, el peso en las niñas, se mueve en un rango de 29,67 hasta $59,83 \mathrm{~kg}$ y, en los niños, oscila entre 31,29 hasta $62,20 \mathrm{~kg}$. La talla en las niñas presenta un rango entre 1,41 hasta $1,61 \mathrm{~cm}$ y, en los niños, los valores se mueven en un rango desde 1,41 hasta $1,65 \mathrm{~cm}$. Los resultados evidencian el crecimiento progresivo del peso y la talla en las edades de 11 a 14 años, más acentuado en los niños que en las niñas.

- El Índice de Masa Corporal se comporta con una tendencia creciente de los 11 a los 14 años, con valores que oscilan, en las niñas, desde 14,86 hasta 24,07 puntos $y$, en los niños, se encuentran en un rango de 15,37 a 22,87 puntos. Los resultados reflejan en promedio valores normales, aunque algunos pocos individuos de la muestra estudiada mostraron valores de sobrepeso.

- Elíndice Córmico es un importanteindicador en los corredores de velocidad, porque unos miembros inferiores largos favorecen el desempeño deportivo. Los resultados en niñas oscilan en un rango de 50,38 hasta $52,63 \%$, ambos valores a los 14 años de edad; esto indica que las niñas presentan tronco corto y tronco intermedio. En los niños, el rango oscila de 49,55, a los 11 años, hasta 51,51 , a los 14 años y también presentan tronco corto y tronco intermedio.

- El porcentaje de grasa de las niñas varía en un rango desde 16,85\%, a los 11 años, hasta $31,77 \%$, a los 14 años. En los niños, los valores 
oscilan entre 13,06 hasta $21,25 \%$, a los 14 años, lo que evidencia, que la grasa corporal aumenta progresivamente en estas edades, tanto en niños como niñas.

- Con frecuencia, se toma como talento a aquel que presenta un desarrollo acelerado y se descartan aquellos con un desarrollo normal o atrasado, desconociendo la edad biológica. Varios autores coinciden en afirmar que los niños con desarrollo atrasado o normal pueden alcanzar y superar los niños con desarrollo acelerado y los que tienen desarrollo acelerado pueden no seguir siendo los mejores. Los profesores y los entrenadores deben considerar la edad biológica, como uno de los factores que influyen en el proceso de aprendizaje motor y rendimiento deportivo.

Conflicto de intereses: Los autores manifiestan no tener conflicto de intereses.

\section{REFERENCIAS}

1. ASHANIN, V.; DRUZ, V.; PYATISOTSKAYA, Y.; ZHERNOVNIKOVA, I.; ALEKSIEIEVA, Y.; ALEKSENKO, A.; YEFREMENKO, O.; PILIPKO, O. 2018. Methods for determining the biological age of different children. Journal of Physical Education and Sport (JPES). 18(4):270.

2. ASHANIN, V.; PYTISOTSKAYA, S.; ZHERNOVNIKOVA, Y.A. 2015. Biological age as a determinating factor of individualization of physical education of children. Ashanin Volodymyr Semenovych. 46(2):221-225. https://doi.org/10.15391/snsv.2015-2.042

3. ASTRAND, P. 2000. Influencias de la edad biológica y de la selección. En: Astrand, P.; Shephard, R. (eds). La resistencia en el deporte. Comité Olímpico Internacional. Editorial. Paidotribo (Barcelona, España). p.305-309.

4. ASTRAND, P.; RODAHL, K. 1992. Fisiología del trabajo físico. Editorial Médica Panamericana (Buenos Aires). 488p.

5. DOLEŽAJOVÁ, L.; GALLOVÁ, T.; LEDNICKÝ, A. 2019. The effect of biological age in the evaluation of physical indicators and the changes in selected motorictests of young female basketball players. Acta Facultatis Education Physicae Universitatis Comenianae. 59(2):118-128. https://doi.org/10.2478/afepuc-2019-0010

6. ELFERINK-GEMSER, M. 2013. Olympia exists pushing boundaries for talented athletes. University of Applied Sciences (Arnhem).

7. EPSTEIN, D. 2014. El gen deportivo. Un atleta excelente ¿nace 0 se hace? Ediciones Urano (Barcelona, España). 382p.

8. HOHMANN, A.; LAMES, M.; LETZEER, M. 2005. Introducción a la ciencia del entrenamiento. Editorial Paidotribo (Barcelona, España). 396p.

9. MALINA, R.; CUMMING, S.; ROGOL, A.; COELHO, M.; FIGUEIREDO, A.; KONARSKI, J.; KOZIEL, S. 2019. BioBanding in Youth Sports: Background, Concept, and Application. Sports Medicine. 49:1671-1685. https://doi.org/10.1007/s40279-019-01166-x

10. MARTÍN, D.; CARL, K.; LEHNERTZ, K. 2001. Manual de metodología del entrenamiento deportivo. Editorial Paidotribo (Barcelona, España). 406p.

11. MILÓ, M. 2007. Propuesta metodológica para la integración del componente edad biológica en el proceso de preparación física de niñas deportistas infantiles de la categoría 11 - 12 años. Tesis doctoral. ISCF Manuel Fajardo (La Habana, Cuba).

12. PANCORBO, A. 2002. Fisiología, Medicina del deporte y ciencias aplicadas al alto rendimiento y la salud. Universidad e Caxias Do Sul. EDUCS.

13. PLATONOV, V. 2001. Teoría general del entrenamiento deportivo olímpico. Editorial Paidotribo (Barcelona, España). 686p.

14. PLATONOV, V.; BULÁTOVA, M. 2015. Selección, orientación, dirección y control en el sistema de preparación de los deportistas. Universidad del Valle y Universidad de Ciencias Aplicadas y Ambientales (Bogotá D.C.). 212p.

15. POLIT, D.; HUNGLER, B. 2000. Investigación científica en las ciencias de la salud. Sexta edición. McGraw Hill - Interamericana (México). 725p. 
16. SIRET, J.; PANCORBO, A.; LOZANO, F.; MOREJÓN, M. 1991. Edad morfológica. Evaluación antropométrica de la edad biológica. Revista cubana de medicina del deporte y la cultura física (La Habana, Cuba). 2(1):7-13.

17. SIRIS,P.;GAIDARSKA,P.;RACHEV,K.1988. Selección y pronóstico de las facultades en el atletismo. Cultura física y deporte (Moscú, Rusia).

18. STEWART, A.; MARFELL-JONES, T.; OLDS, T.; RIDDER, H. 2011. Protocolo internacional para la valoración antropométrica. International Society for the Advancement of Kinanthropometry ISAK (Portsmouth, UK). 126p.

19. SUSLOV, F. 2008. Problemas actuales en el desarrollo de jóvenes atletas. Nuevos Estudios en Atletismo, IAAF (Santa Fe). 7(3):21-27.
20. TITTEL, K.; WUTSCHERK, H. 2000. Fundamentos anatómicos y antropométricos de la resistencia. En: Astrand, P.; Shephard, R. La resistencia en el deporte. Editorial Paidotribo (Barcelona, España). p.36-47.

21. VOLKOV, V.; FILIN, V. 1988. Selección deportiva. Cultura física y deporte (Moscú, Rusia).

22. WEINECK, J. 2012. Descubrimiento de talentos y velocidad. Conferencia impartida en lugar no establecido.

23. WUTSCHERK, H. 1974. Bestimmung des biologischen Alters. Theorie und Praxis der Körperkultur. 23:159-170

24. ZATSIORSKI, V. 1989. Metrología deportiva. Editorial Planeta (Moscú, Rusia). 298p. 